\title{
Two cases of cardiac glycoside poisoning from accidental foxglove ingestion
}

\author{
Renée M. Janssen MD MSc, Mattias Berg MD, Daniel H. Ovakim MD MSc
}

A 67-year-old Chinese woman presented to the emergency department with a three-day history of nausea and vomiting, palpitations and chest discomfort. Her symptoms had started suddenly a few hours after eating lunch, beginning with generalized weakness and fatigue; she had noticed that her pulse was slow and irregular. A few hours later, chest pressure developed along with abdominal cramping associated with nausea and vomiting, which persisted.

The patient's medical history included hypertension, dyslipidemia, Meniere disease and asthma. She was not taking prescription medications; supplements included vitamin $\mathrm{B}_{12}$, glucosamine and Chinese herbs (prepared licorice, dried rehmannia root, cinnamon twig, Ophiopogon japonicus, ejiao, hemp seed, jujube and ginger). Her vital signs were notable for bradycardia (53 beats/min); other findings on physical examination were normal.

Results of initial laboratory investigations, including electrolyte levels, renal function, liver biochemistry and synthetic function, were normal. Her serum potassium level was 4.1 (normal 3.55.0) $\mathrm{mmol} / \mathrm{L}$ and magnesium level 0.96 (normal 0.70-1.10) mmol/L. Complete blood count revealed a slightly elevated leukocyte count $(14.0 \times$ $\left.10^{9} / \mathrm{L}\right)$. Serial troponin levels were not elevated. A chest radiograph was normal. An electrocardiogram (ECG) showed sinus rhythm with firstdegree atrioventricular block and sinus pauses of one to two seconds. The patient was admitted to the cardiac intensive care unit for monitoring.

Over the next four days, recurrent dysrhythmias developed, including third-degree atrioventricular block with accelerated junctional rhythm, sinus bradycardia, frequent premature ventricular contractions, nonspecific ST-segment changes and $\mathrm{T}$-wave inversion in the lateral leads. On day 4, a 30-second episode of monomorphic ventricular tachycardia occurred (Figure 1) that prompted consideration for insertion of an implantable cardioverter-defibrillator. Coronary angiography showed no substantial coronary artery disease, and transthoracic echocardiography showed normal biventricular structure and function.

On day 3, the patient's husband, age 66, presented to the same emergency department with similar symptoms. He had a medical history of dyslipidemia and was taking no prescription medications. Laboratory investigations showed normal electrolyte levels (potassium $4.3 \mathrm{mmol} / \mathrm{L}$, magnesium $1.10 \mathrm{mmol} / \mathrm{L}$ ), renal function and complete blood count. Serial troponin levels were not elevated. An ECG showed sinus bradycardia with nonspecific ST-segment changes and lateral T-wave inversion; there was no previous ECG for comparison. In the emergency department, the husband was noted to have episodes of bradycardia, with a heart rate in the $30 \mathrm{~s}$. Both patients reported visual disturbances, described as flashing lights and yellow/red halos around objects.

On further questioning, the wife revealed they had consumed a bitter-tasting leafy green plant on the day her symptoms began. Her husband had picked the leaves from their vegetable garden, believing them to be kale. Given the constellation of symptoms in both patients following this meal, a toxic ingestion seemed likely.

Serum digoxin levels, normally undetectable, were $0.3 \mathrm{nmol} / \mathrm{L}$ in the wife and $0.4 \mathrm{nmol} / \mathrm{L}$ in the husband. The family brought in plant specimens from their garden (Figure 2), which were later confirmed to be foxglove (Digitalis purpurea).
Competing interests: None declared.

This article has been peer reviewed.

The authors have obtained patient consent.

Correspondence to: Renée Janssen, renee.janssen@ alumni.ubc.ca

CMAJ 2016. DOI:10.1503 /cmaj.150676 
Once the diagnosis of cardiac glycoside toxicity was confirmed and the provincial poison control centre was consulted, both patients were given digoxin-Fab. The husband initially received 5 vials, and his wife, whose symptoms were more severe, received 10 vials. Both patients experienced considerable improvement in symptoms within an hour after receiving the antidote. Over the course of the next several days, they were each administered a total of 12 vials. Both patients experienced complete resolution of their symptoms and were discharged home.

\section{Discussion}

Foxglove (including D. purpurea and Digitalis lanata) is one of many plants containing cardiac glycosides that exert potent inotropic and electrochemical effects on cardiac tissue. Hun- dreds of cardiac glycosides have been isolated from plants across 12 botanical families, including members of the Digitalis genus (foxglove), Cascabela thevetia (yellow oleander) and Convallaria majalis (lily of the valley). The cardiac glycosides of medical importance are extracted from $D$. purpurea and D. lanata, yielding digitoxin and digoxin, respectively.

Foxglove (Figure 3) is native to Europe and widely naturalized to temperate climes in North America, including Canada. Accidental ingestion of foxglove is uncommon because of its distinctive flowers and bitter taste. However, the plant does not flower in its first year, and the rosette of basal leaves of the juvenile plant has been mistaken for borage $^{1}$ or comfrey. ${ }^{2}$ In British Columbia, the two cases we have described here constitute the only cases of symptomatic cardiac glycoside poisoning from plant ingestion requiring antidote

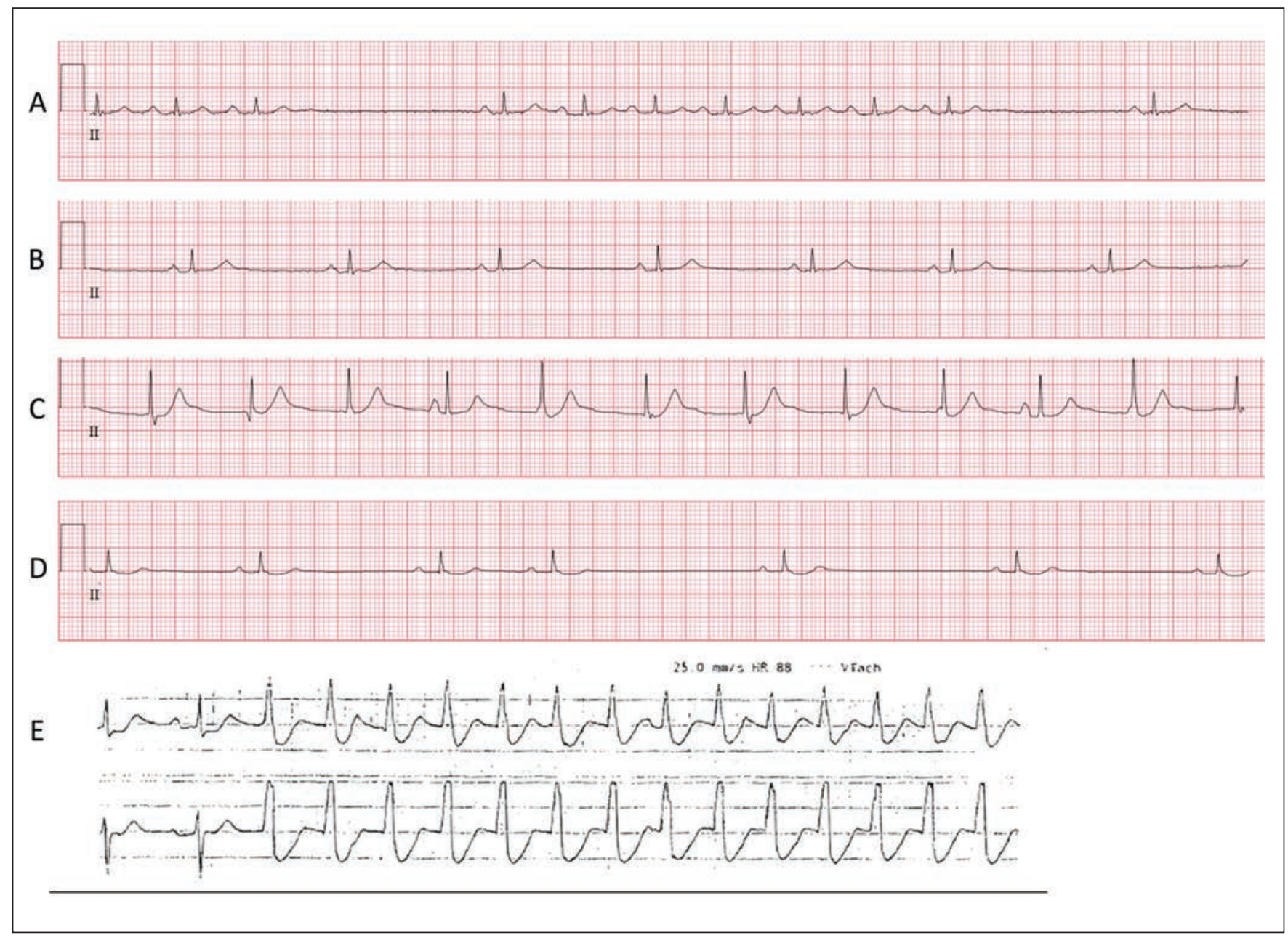

Figure 1: Electrocardiogram (ECG) strips from lead II (A through D) and leads II and V1 (E), showing the progression of ECG changes in a 67-year-old woman during her hospital admission, before administration of digoxin immune antibody fragments. (A) Admission ECG shows sinus rhythm with first-degree atrioventricular block (PR interval $210 \mathrm{~ms}$ ) and sinus pauses. (B) ECG later on day 1 shows sinus bradycardia (heart rate 45 beats/min); PR interval has returned to normal (166 ms). (C) ECG on day 2 shows accelerated junctional rhythm with P waves and atrioventricular dissociation (complete heart block). (D) ECG on day 3 shows sinus bradycardia with nonspecific ST-segment changes and ST-segment depression. (E) ECG from telemetry strip on day 4 of admission shows monomorphic ventricular tachycardia, which lasted for about 30 seconds and was self-limited. 
treatment since 2012. Toxicity from pharmaceutical digoxin use is much more common, with 184 cases receiving antidotal treatment in the province from 2012 to 2014 (Dr. Debra Kent, BC Drug and Poison Information Centre: personal communication, 2015).

\section{Manifestations of toxicity}

Cardiac glycosides exert their effects on myocardial tissue by inhibiting the sodium-potassium-adenosinetriphophatase (Na-K-ATPase) enzyme, leading to increased intracellular sodium and calcium concentrations; this effect is responsible for both the desired positive inotropic effects and the undesirable arrhythmias. Cardiac glycosides are also believed to exert effects on the autonomic nervous system, causing suppression of sympathetic activation and increased vagal tone.

The cardiac manifestations of cardiac glycoside toxicity result from increased automaticity with concomitant conduction block. Although almost any arrhythmia may result, paroxysmal atrial tachycardia with block, accelerated junctional rhythm and bidirectional ventricular tachycardia are highly suggestive of cardiac glycoside toxicity. ${ }^{3}$ Death typically results from refractory ventricular fibrillation or asystole. The classic ECG findings of T-wave changes, QTinterval shortening, ST-segment scooping and increased U-wave amplitude, collectively known as the "digitalis effect," reflect electrochemical changes that result from the presence of digitalis and do not indicate toxicity. ${ }^{3}$

Systemic symptoms resulting from cardiac glycoside intoxication include nausea, vomiting, abdominal pain, anorexia, visual disturbances, confusion, weakness, lethargy and delirium (Box 1). ${ }^{4}$

There are variable effects on the serum potassium level. In acute toxicity, hyperkalemia (serum potassium $>5.5 \mathrm{mmol} / \mathrm{L}$ ) results from blockade of the Na-K-ATPase pump in both cardiac tissue and skeletal muscle and is a marker of severe toxicity. Conversely, hypokalemia is more common in chronic toxicity and confers increased susceptibility to dysrhythmias at lower glycoside concentrations. ${ }^{5}$

At the time of admission, both patients presented with normokalemia, despite the severity of their other symptoms. Their normal potassium values may have been due the late presentation following several days of vomiting. In addition, the wife was taking licorice extract, which is known to cause hypokalemia through inhibition of 11- $\beta$-hydroxysteroid dehydrogenase. None of the herbs that she was taking contained cardiac glycosides and likely did not contribute to her symptoms.
Pharmaceutical cardiac glycoside toxicity Cardiac glycosides, such as digoxin, have long been used to improve inotropy in patients with congestive heart failure, and to slow ventricular rates in patients with atrial fibrillation or flutter. Although the therapeutic range for digoxin is $0.64-1.28 \mathrm{nmol} / \mathrm{L}$, cardiotoxicity has been reported in patients maintained within this range, particularly those receiving long-term treatment. Serum digoxin levels, and the risk of subsequent toxicity, are highly susceptible to changes in renal function, serum potassium levels (hypokalemia), concurrent use of medications that interfere with digoxin excretion (e.g., amiodarone and non-dihydropyridine calcium-channel blockers) and genetic polymorphisms that impair the activity of P-glycoprotein. ${ }^{6}$ Digoxin toxicity should be suspected and treated in patients who present with consistent clinical features regardless of their serum digoxin level (Box 2).

\section{Diagnosis}

Cardiac glycoside toxicity is primarily a clinical diagnosis. Serum digoxin levels should be obtained to confirm the diagnosis and to guide antidote administration (in the context of pharmaceutical digoxin poisoning). ${ }^{8}$ Although there is crossreactivity with other cardiac glycosides, the digoxin immunoassay is specific for the detection of digoxin. Because of unpredictable cross-reactivity, the quantitative value has no relation to the degree of toxicity from exposure to nonpharmaceutical cardiac glycosides, and may even be undetectable. ${ }^{8}$ The serum digoxin level should therefore not be used to guide antidote dosage in cases of nonpharmaceutical cardiac glycoside ingestion.

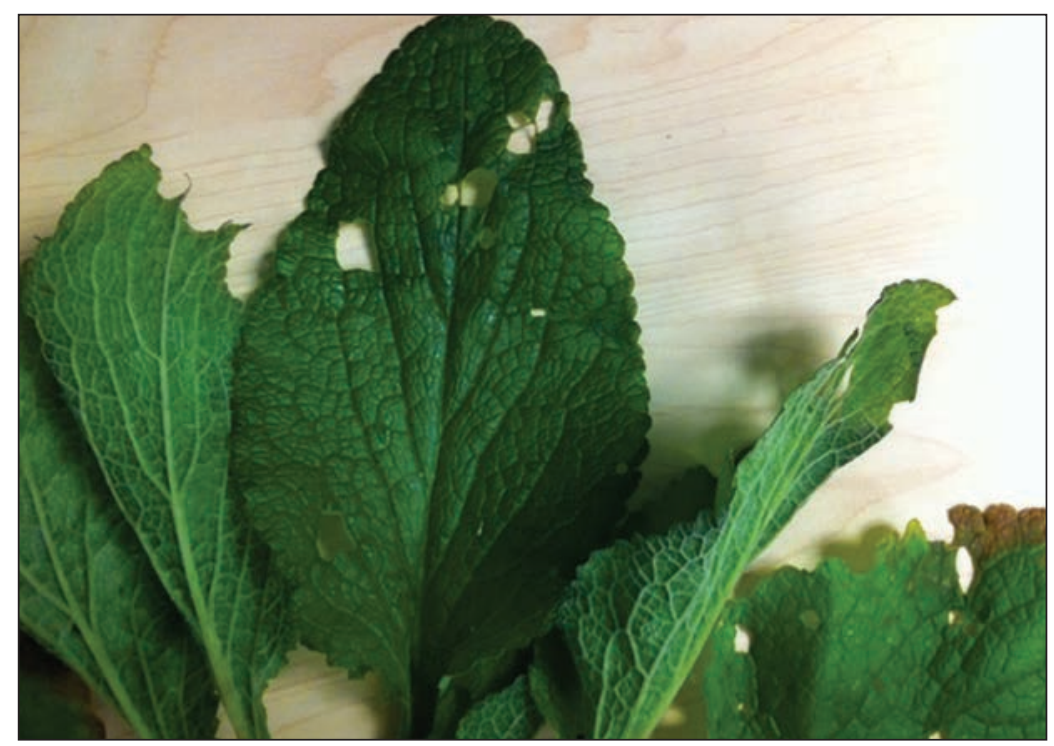

Figure 2: A plant specimen from the family's garden that the husband and wife consumed before presentation to hospital. Subsequent analysis confirmed the plant to be foxglove (Digitalis purpurea). 


\section{Management}

The management of cardiac glycoside poisoning includes supportive care and antidote therapy with digoxin-specific antibody fragments (digoxinFab). Digoxin-Fab is indicated for severe cases of

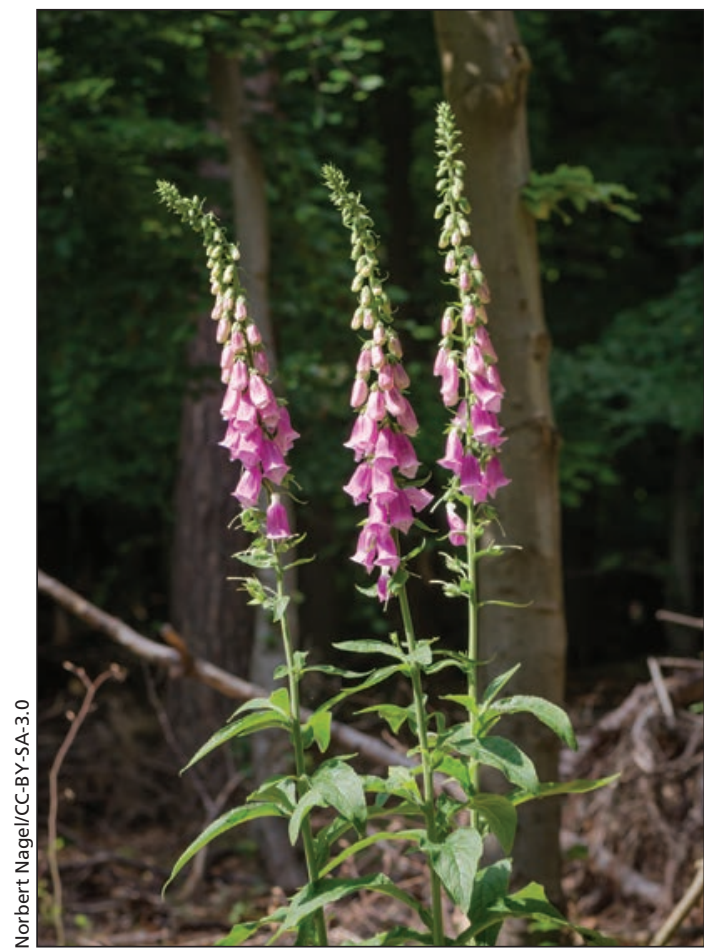

Figure 3: Mature foxglove plants (Digitalis purpurea) in bloom in late spring.

\section{Box 1: Signs and symptoms of acute and chronic cardiac glycoside} toxicity ${ }^{4}$

- General: Fatigue, malaise, generalized weakness

- Gastrointestinal: Nausea, vomiting, anorexia, abdominal pain, diarrhea

- Cardiovascular: Dysrhythmias, palpitations, chest pain

- Central nervous system: Confusion, delirium, seizures, psychosis, abnormal dreams, colour vision disturbance (xanthopsia)

- Electrolytes: Hyperkalemia (acute toxicity) or hypokalemia (chronic toxicity)

\section{Box 2: Indications for digoxin-specific antibody fragments?}

- Any potentially life-threatening dysrhythmia related to cardiac glycosides, including:

- ventricular tachycardia

- ventricular fibrillation

- progressive bradycardia

- second- or third-degree heart block not responsive to atropine

- Serum digoxin level $>12.8 \mathrm{nmol} / \mathrm{L}$ measured more than 6 hours after ingestion in an acute ingestion

- Acute ingestion of $>10 \mathrm{mg}$ in an adult (4 mg in a child)

- Serum potassium level $>5.5 \mathrm{mmol} / \mathrm{L}$ in an acute ingestion

- Ingestion of nonpharmaceutical cardiac glycoside (e.g., as found in plants, insects) cardiac glycoside toxicity (Box 2). It should be avoided in milder cases because of the theoretical risk of immunogenicity. Currently available commercial preparations include DigiFab and DigiBind (the latter of which is unavailable in North America); each vial, administered intravenously, binds $0.5 \mathrm{mg}$ of digoxin. ${ }^{7}$ Following administration of digoxin-Fab, serum total digoxin levels should not be measured until the antidote has been eliminated from the body (up to three weeks in patients with impaired kidney function), due to interference with the immunoassay. ${ }^{7}$

Digoxin-Fab has also proven to be effective as an antidote for poisonings from other cardiac glycosides. A randomized controlled trial involving 66 patients with cardiac glycoside toxicity from ingestion of yellow oleander in Sri Lanka found that digoxin-Fab resulted in a significant resolution of dysrhythmias and hyperkalemia compared with saline placebo. ${ }^{9}$ Several other observational studies have reported favourable outcomes with use of digoxin-Fab in cases of plant-related cardiac glycoside poisonings. ${ }^{10}$ Dosing recommendations for symptomatic nondigoxin cardiac glycoside exposure suggest empiric administration of 10 vials of digoxin-Fab. ${ }^{7}$

\section{References}

1. Maffè S, Cucchi L, Zenone F, et al. Digitalis must be banished from the table: a rare case of acute accidental digitalis intoxication of a whole family. J Cardiovasc Med (Hagerstown) 2009; 10:727-32.

2. Lin CC, Yang CC, Phua DH, et al. An outbreak of foxglove leaf poisoning. J Chin Med Assoc 2010;73:97-100.

3. Ma G, Brady WJ, Pollack M, et al. Electrocardiographic manifestations: digitalis toxicity. J Emerg Med 2001;20:145-52.

4. Mahdyoon H, Battilana G, Rosman $\mathrm{H}$, et al. The evolving pattern of digoxin intoxication: observations at a large urban hospital from 1980 to 1988. Am Heart J 1990;120:1189-94.

5. Eddleston M, Ariaratnam CA, Sjöström L, et al. Acute yellow oleander (Thevetia peruviana) poisoning: cardiac arrhythmias, electrolyte disturbances, and serum cardiac glycoside concentrations on presentation to hospital. Heart 2000;83:301-6.

6. Xu P, Jiang ZP, Zhang BK, et al. Impact of MDR1 haplotypes derived from C1236T, G2677T/A and C3435T on the pharmacokinetics of single-dose oral digoxin in healthy Chinese volunteers. Pharmacology 2008;82:221-7.

7. DigiFab [product monograph]. Ottawa: Canadian Pharmacists Association. Available: www.e-cps.ca (accessed 2015 May 11).

8. Smith TW, Antman EM, Friedman PL, et al. Digitalis glycosides: mechanisms and manifestations of toxicity. Part I. Prog Cardiovasc Dis 1984;26:413-58.

9. Eddleston M, Rajapakse S, Rajakanthan K, et al. Anti-digoxin $\mathrm{Fab}$ fragments in cardiotoxicity induced by ingestion of yellow oleander: a randomised controlled trial. Lancet 2000;355:967-72.

10. Roberts DM, Buckley NA. Antidotes for acute cardenolide (cardiac glycoside) poisoning. Cochrane Database Syst Rev 2006; (4):CD005490.

Affiliations: Departments of Medicine (Janssen, Ovakim) and Emergency Medicine (Berg), University of British Columbia; British Columbia Drug and Poison Information Centre (Ovakim), Vancouver, BC

Contributors: Renée Janssen drafted the manuscript. All of the authors contributed equally to the revising of the manuscript, gave final approval of the version to be published and agreed to act as guarantors of the work. 